\title{
On Analog of Fourier Transform in Interior of the Light Cone
}

\author{
Tatyana Shtepina \\ Donetsk Institute of Municipal Economy, Bulavina Street 1, Donetsk 83053, Ukraine \\ Correspondence should be addressed to Tatyana Shtepina; tatyana.shtepina@gmail.com
}

Received 7 November 2013; Revised 6 May 2014; Accepted 7 May 2014; Published 6 August 2014

Academic Editor: Adem Kilicman

Copyright (C) 2014 Tatyana Shtepina. This is an open access article distributed under the Creative Commons Attribution License, which permits unrestricted use, distribution, and reproduction in any medium, provided the original work is properly cited.

We introduce an analog of Fourier transform $\mathscr{F}_{h}^{\rho}$ in interior of light cone that commutes with the action of the Lorentz group. We describe some properties of $\mathscr{F}_{h}^{\rho}$, namely, its action on pseudoradial functions and functions being products of pseudoradial function and space hyperbolic harmonics. We prove that $\mathscr{F}_{h}^{\rho}$-transform gives a one-to-one correspondence on each of the irreducible components of quasiregular representation. We calculate the inverse transform too.

\section{Introduction}

One of the most valuable integral transforms used in manydimensional analysis is the classical Fourier transform. It is caused by the fact that this transform has a very simple transformation law at tensions and commutes with action of Lie group $S O(n)$ in $\mathbb{R}^{n}$. As a consequence of these properties, the $L^{2}\left(\mathbb{R}^{n}\right)$ decomposes in a direct sum of irreducible subspaces that are invariant under rotations (e.g., [1, chapter 4] and [2, chapter 9]). This decomposition is an analog of decomposition $L^{2}(\mathbb{R})$ in a direct integral of irreducible representations:

$$
L^{2}(\mathbb{R})=\int_{-\infty}^{\infty} \mathbb{C} \cdot e^{i x \xi} d \xi,
$$

which act in one-dimensional subspaces invariant under translations. There exists a general theorem that guarantees the existence of a direct integral decomposition into irreducible subrepresentations: it suffices that the topological group have a countable dense subset.

The goal of this paper is to introduce an analog of Fourier transform $\mathscr{F}_{h}^{\rho}$ in the interior of the light cone on which Lie group $G=S O_{0}(n-1,1)$ acts. Suppose $L_{\text {loc }}^{1}\left(S_{H}(R)\right)$ is a space of locally integrable functions on pseudosphere $S_{H}(R)$ of radius $R$, so this space allows a direct integral decomposition into irreducible subspaces invariant under action of Lie group $G$ that is similar to decompositions of $L^{2}\left(\mathbb{R}^{n}\right)$ and $L^{2}(\mathbb{R})$ in classical case. Actually, this decomposition was obtained by Gel'fand et al. in [3] in the sixties of the last century.
Our analog of Fourier transform is an intertwining operator of quasiregular representation of Lie group $G$, so it maps each of the irreducible components of decomposition in itself. Following Stein and Weiss in Euclidean space [1], we describe action of $\mathscr{F}_{h}^{\rho}$ on pseudoradial functions and functions that represent a product of pseudoradial function and space hyperbolic harmonics. The obtained formulas allow us to write the inverse transform $\left(\mathscr{F}_{h}^{\rho}\right)^{-1}$ with ease. These results may be applicable to constructing an equivariant extension of wave operator in interior of the light cone. For Laplace operator it was completed in [4].

\section{Spherical Harmonics and Classical Funk-Hecke Theorem}

Let $\mathfrak{R}^{n, l}$ be the space of homogeneous harmonic polynomials of degree $l$ in $n$ variables. If $f(x)$ belongs to $\mathfrak{R}^{n, l}$ then its restriction to sphere $S^{n-1}$ is called the surface spherical harmonics of degree $l$ and is denoted by $f(\xi), \xi \in S^{n-1}$. The relation between $f(x)$ and $f(\xi)$ follows from homogeneity condition:

$$
f(x)=f(r \cdot \xi)=r^{l} f(\xi), \quad r=|x| .
$$

Surface spherical harmonics of degree $l$ form a linear space over $\mathbb{C}$ too, and we denote it by $\mathscr{R}^{n, l}$. It is quite evident that, for any $l=0,1,2, \ldots$, the inclusion $\mathscr{R}^{n, l} \subset L^{2}\left(S^{n-1}\right)$ is valid. But in 
the same space acts the so-called quasiregular representation of Lie group $S O(n)$, defined by the next equality:

$$
[T(g) f](\xi)=f\left(g^{-1} \xi\right), \quad \xi \in S^{n-1}, g \in S O(n) .
$$

This representation is unitary with respect to the standard inner product in $L^{2}\left(S^{n-1}\right)$. The next theorem is widely known (see, e.g., $[2,5,6])$.

Theorem 1. (a) The next decomposition is valid $L^{2}\left(S^{n-1}\right)=$ $\bigoplus_{l=0}^{\infty} \mathscr{R}^{n, l}$ (decomposition of Hilbert space into orthogonal direct sum).

(b) The subspaces $\mathfrak{R}^{n, l}$ consisting of the space harmonics of degree $l$ are invariant under Fourier transform $\mathscr{F}$.

(c) Each of the subspaces $\mathscr{R}^{n, l}$ is invariant with respect to the quasiregular representation $T$ of Lie group $S O(n)$ and is isomorphic to the irreducible representation $T_{(l, 0, \ldots, 0)}$ with a highest weight $(l, 0, \ldots, 0)$.

(d) The quasiregular representation of $S O(n)$ in $L^{2}\left(S^{n-1}\right)$ has a simple spectrum.

(e) The space $\mathscr{R}^{n, l}$ has a dimension $(n+l-3) !(n+2 l-$ $2) / l !(n-2)$ ! and an orthogonal basis consisting of the next surface spherical harmonics $S_{K}^{l}(\theta)$ :

$$
\begin{aligned}
S_{K}^{l}(\theta)= & \left(\prod_{j=0}^{n-3} C_{m_{j}-m_{j+1}}^{n / 2-j / 2-1+m_{j+1}}\left(\cos \theta_{n-j-1}\right)\right. \\
& \left.\times \sin ^{m_{j+1}}\left(\theta_{n-j-1}\right)\right) \\
& \times e^{ \pm i m_{n-2} \theta_{1}},
\end{aligned}
$$

where $\theta_{1}, \theta_{2}, \ldots, \theta_{n-1}$ are Euler angles on sphere $S^{n-1} ; l=m_{0}$; and $K$ is multi-index $K=\left(m_{1}, \ldots, m_{n-3} ; \pm m_{n-2}\right)$ such that $m_{0} \geq m_{1} \geq \cdots \geq m_{n-3} \geq m_{n-2} \geq 0, m_{i} \in \mathbb{Z}$.

The known Funk-Hecke theorem states that for integral operators whose kernels depend only on the distance $\rho$ (in spherical geometry) between points $\xi$ and $\eta$ where $\xi, \eta \in$ $S^{n-1}$ every surface spherical harmonics is an eigenvector. We give a contemporary formulation of the Funk-Hecke theorem following the monograph [7] of Erdélyi.

Theorem 2 (Funk, Hecke). Let $F(x)$ be a function of a real variable $x$ which is absolutely Lebesgue integrable on $[-1,1]$ together with its square. Then, for any unit vector $\eta$,

$$
\int_{S^{n-1}} F[(\xi, \eta)] S_{K}^{l}(\xi) d \xi=\lambda_{n, l} S_{K}^{l}(\eta)
$$

where

$$
\begin{gathered}
\lambda_{n, l}=i^{l}(2 \pi)^{n / 2} \int_{-\infty}^{\infty} t^{(2-n) / 2} J_{l+n / 2-1}(t) f(t) d t, \\
f(t)=\frac{1}{2 \pi} \int_{-1}^{1} e^{-i x t} F(x) d x .
\end{gathered}
$$

The simple consequences of the Funk-Hecke theorem are the following two propositions.
Proposition 3 (see [1, chapter IV, Theorems 3.3, 3.10]). (a) Let function $f(x)$ be a product of radial function and space spherical harmonics of degree $l$ :

$$
f(x)=f_{0}(|x|) S_{K}^{l}(x),
$$

where $f_{0}(r)$ is such that $f(x) \in L^{1}\left(\mathbb{R}^{n}\right) \cap L^{2}\left(\mathbb{R}^{n}\right)$. Then its Fourier transform has a form:

$$
[\mathscr{F} f](x)=F_{l}(|x|) S_{K}^{l}(x),
$$

where

$$
\begin{aligned}
F_{l}(r)= & \frac{2 \pi}{i^{l} r^{(n-2) / 2+l}} \\
& \times \int_{0}^{+\infty} f_{0}(s) s^{l+n / 2} J_{(n+2 l-2) / 2}(2 \pi r s) d s .
\end{aligned}
$$

(b) In particular, Fourier transform for radial function $f(x)=$ $f_{0}(|x|)$ is also radial:

$$
[\mathscr{F} f](x)=F_{0}(|x|)
$$

(one sets $l=0$ in the above formula).

As Proposition 3 implies, the infinite-dimensional subspaces

$$
\mathscr{H}_{l}=\operatorname{span}\left\{f(|x|) S_{K}^{l}(x)\right\}
$$

where $f(r)$ runs over the set of radial functions satisfying conditions of Proposition 3 and $S_{K}^{l}(x)$ runs over the set of space spherical harmonics of degree $l$, are invariant under Fourier transform in $L^{2}\left(\mathbb{R}^{n}\right)$.

On the other hand, if we fix the function $f(|x|)$ we get a subspace in $\mathscr{H}_{l}$, which is invariant with respect to quasiregular representation of group $S O(n)$ in $L^{2}\left(\mathbb{R}^{n}\right)$. It can be easily verified that it is isomorphic to the irreducible representation $T_{(l, 0, \ldots, 0)}$ with a highest weight $(l, 0, \ldots, 0)$. Since spaces of irreducible nonisomorphic unitary representations of compact group are mutually orthogonal $(\mathrm{H}$. Weyl's theorem), we have one more important consequence of the classical Funk-Hecke theorem.

Corollary 4. The next decomposition into orthogonal direct sum is valid:

$$
L^{2}\left(\mathbb{R}^{n}\right)=\bigoplus_{l=0}^{\infty} \mathscr{H}_{l}
$$

We will try to extend the classical theorem of Funk and Hecke and its corollaries on the hyperbolic space $\mathbb{R}^{n-1,1}$ with indefinite inner product.

\section{Hyperbolic Harmonics and Generalized Funk-Hecke Theorem}

Let $\mathbb{R}^{n-1,1}$ be the pseudo-Euclidean space with the indefinite inner product

$$
[x, y]=-x_{1} y_{1}-\cdots-x_{n-1} y_{n-1}+x_{n} y_{n}
$$


This inner product may be used for definition of a distance $r(x, y)$ between two points $x, y \in \mathbb{R}^{n-1,1}$ that do not belong to the light cone $[z, z]=0$. We assume, for such two points,

$$
\cosh r(x, y)=\frac{[x, y]}{\sqrt{[x, x] \cdot[y, y]}} .
$$

Such distance may take either real nonnegative or pure imaginary values. However, if we restrict ourselves by the interior $U$ of the light cone's upper sheet

$$
U=\left\{x \in \mathbb{R}^{n-1,1} \mid[x, x]>0, x_{n}>0\right\},
$$

then, for all $x, y \in U$, we have $r(x, y) \geqslant 0$.

Let us call the set of all points $x$ of $U$, for which $[x, x]=R^{2}$ holds, pseudosphere of radius $R$. We will use the designation $S_{H}(R)$ for pseudosphere of radius $R$ and $S_{H}$ for pseudosphere of radius 1 in $\mathbb{R}^{n-1,1}$. Recall that $S_{H}$ is a manifold of a constant negative curvature in $\mathbb{R}^{n-1,1}$ on the one hand and a homogeneous symmetric space with respect to the action of Lie group $G=S O_{0}(n-1,1)$ on the other hand, because

$$
S_{H} \cong S O_{0}(n-1,1) / S O(n-1) .
$$

It follows from here that $S_{H}$ possesses the unique up to constant multiplier left-invariant with respect to $G$ measure $d \xi$ :

$$
d \xi=\sinh ^{n-2} \theta_{n-1} \sin ^{n-3} \theta_{n-2} \cdot \ldots \cdot \sin \theta_{2} d \theta_{1} d \theta_{2} \cdots d \theta_{n-1} .
$$

We denote by $L_{\text {loc }}^{1}\left(S_{H}, d \xi\right)$ the space of complex-valued functions on $S_{H}$ locally integrable in measure $d \xi$. In $L_{\text {loc }}^{1}\left(S_{H}, d \xi\right)$ acts the quasiregular representation $R$ of Lie group $G$, defined by

$$
(R(g) f)(x)=f\left(g^{-1} x\right), \quad x \in S_{H}, g \in S_{0}(n-1,1) .
$$

We need the notion of space and surface hyperbolic harmonics to decompose the representation $R$ into irreducible ones. We will consider in $L_{\text {loc }}^{1}\left(S_{H}, d \xi\right)$ functions

$$
\begin{aligned}
H_{L}^{n, \sigma}(\theta)= & \sinh ^{(3-n) / 2} \theta_{n-1} \mathfrak{P}_{\sigma+(n-3) / 2}^{(3-n) / 2-m_{1}}\left(\cosh \theta_{n-1}\right) \\
& \times S_{K}^{m_{1}}\left(\theta_{1}, \theta_{2}, \ldots, \theta_{n-2}\right),
\end{aligned}
$$

where $\mathfrak{P}_{\nu}^{\mu}(x)$ are adjoined Legendre functions of genus one, $L=\left(k_{0}, K\right), K=\left(k_{1}, \ldots, k_{n-4}, \pm k_{n-3}\right)$, with $k_{0} \geqslant k_{1} \geqslant \cdots \geqslant$ $k_{n-2} \geqslant 0$, and all parameters $k_{i}$ are integers.

It is easy to see that if we extend functions $H_{L}^{n, \sigma}(\theta)$ from pseudosphere $S_{H}$ to the interior $U$ of the light cone's upper sheet "by homogeneity" with the degree

$$
\sigma=-\frac{n-2}{2}+i \rho, \quad \rho \in[0,+\infty)
$$

then obtained functions $\widetilde{H}_{L}^{n, \sigma}$ on $U$ are solutions of the wave equation $\square \widetilde{H}_{L}^{n, \sigma}=0$; that is, they are space hyperbolic harmonics. This means that we may call $H_{L}^{n, \sigma}(\theta)$ surface hyperbolic harmonics and consider them analogs of surface spherical harmonics $S_{K}^{l}(\theta)$.

The relation between $H_{L}^{n \sigma}$ and $\widetilde{H}_{L}^{n, \sigma}$ follows from homogeneous condition:

$$
\widetilde{H}_{L}^{n, \sigma}(u)=\widetilde{H}_{L}^{n, \sigma}(r \cdot x)=r^{\sigma} \widetilde{H}_{L}^{n, \sigma}(x), \quad r=|x| .
$$

Suppose $\mathscr{G}^{n, \sigma}$ is the minimal closed subspace in $L_{\text {loc }}^{1}\left(S_{H}, d \xi\right)$ containing all surface hyperbolic harmonics $H_{L}^{n, \sigma}(\theta)$. Similarly to Euclidean case, denote by $\mathfrak{G}^{n, \sigma}$ the minimal closed subspace in $L_{\text {loc }}^{1}(U, d u)$ containing all space hyperbolic harmonics. It is obvious, from what is stated above, that the $H_{L}^{n, \sigma}(\theta)$ are linearly independent for different $\sigma$ and all of them are subspaces in the space of wave equation solutions.

Basic properties of $\mathscr{G}^{n, \sigma}$ are proved in [6]. We formulate them in a compact form now.

Theorem 5 (analog of Theorem 1). (a) The next decomposition is valid $L_{l o c}^{1}\left(S_{H}, d \xi\right)=\int_{0}^{\infty} \mathscr{G}^{n, \sigma} d \rho$ (the decomposition into continuous direct sum).

(b) Each of the subspaces $\mathscr{G}^{n, \sigma}$ is invariant with respect to the quasiregular representation $R$ of Lie group $\mathrm{SO}_{0}(n-1,1)$.

(c) The representations of $\mathrm{SO}_{0}(n-1,1)$ in $\mathscr{G}^{n, \sigma}$ are irreducible and mutually nonisomorphic.

(d) The quasiregular representation $R$ of $G$ in $L_{l o c}^{1}\left(S_{H}, d \xi\right)$ has a simple spectrum.

(e) The space $\mathscr{G}^{n, \sigma}$ is infinite-dimensional and has a basis generated by functions of the form $H_{L}^{n, \sigma}(\theta)$.

The following theorem generalizes the classical FunkHecke theorem to the case of hyperbolic space. This theorem, for cases $n=2$ and $n=4$, was proved in [8]. But the general case was published in [6].

Theorem 6. Suppose $F(x)$ is a function of a real variable $x$ such that

(a) $F(x) \in L^{1}(-\infty,+\infty) \cap L^{2}(-\infty,+\infty)$;

(b) $F(x)$ can be continued analytically to a function $F(\alpha)$ of the complex variable $\alpha=x+i y$ that is bounded and analytic in the lower half-plane $y \leqslant 0$;

(c) $F(x)$ has Fourier preimage $f(t) \in L^{1}(0,+\infty)$.

Let $H_{L}^{n, \sigma}$ be an arbitrary surface hyperbolic harmonic of homogeneity degree $\sigma$. Then, for any vector $\eta \in S_{H}$, the following equality holds:

$$
\int_{S_{H}} F([\xi, \eta]) H_{L}^{n, \sigma}(\xi) d \xi=\lambda_{n, \sigma} H_{L}^{n, \sigma}(\eta),
$$

where the eigenvalue $\lambda_{n, \sigma}$ does not depend on index $L$ of harmonics $H_{L}^{n, \sigma}$ and equals

$$
\begin{aligned}
\lambda_{n, \sigma}= & 2^{n / 2}(-\pi i)^{(n-2) / 2} \\
& \times \int_{0}^{+\infty} \alpha^{2-n / 2} K_{\sigma+n / 2-1}(i \alpha) f(\alpha) d \alpha,
\end{aligned}
$$


where $K_{v}(z)$ is the McDonald function and

$$
f(\alpha)=\frac{1}{\sqrt{2 \pi}} \int_{-\infty}^{+\infty} e^{i \alpha t} F(t) d t .
$$

The idea of the proof lies in using of intertwining operators theory. Namely, let us define an operator $\mathscr{A}$ in $L_{\text {loc }}^{1}\left(S_{H}\right)$ by the equality

$$
[\mathscr{A} f](\eta)=\int_{S_{H}} F[(\xi, \eta)] f(\xi) d \xi .
$$

It can be easily seen that $\mathscr{A}$ is an intertwining operator of quasiregular representation $R$. Because the spectrum of $R$ is simple, $\mathscr{A}$ can map the invariant subspace $\mathscr{G}^{n, \sigma}$ only to itself. Schur's lemma implies that $\left.\mathscr{A}\right|_{\mathscr{g}^{n, \sigma}}=\lambda_{n, \sigma} \cdot E$, where $\lambda_{n, \sigma}$ does not depend on the multi-index $L$ and $E$ is identity operator.

Thus one can assume that $L=(0,0, \ldots, 0)$; that is, examine the zonal hyperbolic harmonics $H_{O}^{n \sigma}(\xi)$ instead of arbitrary spherical harmonics $H_{L}^{n \sigma}(\xi)$.

The nontrivial part of the proof lies in calculation of eigenvalue $\lambda_{n, \sigma}$ rather than in verifying if surface hyperbolic harmonics are eigenvectors for $\mathscr{A}$.

\section{The Hyperbolic Fourier Transform and Some of Its Properties}

To obtain analogs of Proposition 3 we need some integral transform $\mathscr{F}_{h}^{\rho}$ in $L_{\text {loc }}^{1}(U)$ similar to the Fourier transform in $L^{2}\left(\mathbb{R}^{n}\right)$.

Definition 7. The hyperbolic Fourier transform in space $L_{\text {loc }}^{1}(U)$ is a transform $\mathscr{F}_{h}^{\rho}$, defined by (this integral should be understood in a regularized value sense)

$$
\left[\mathscr{F}_{h}^{\rho} f\right](\xi)=|\xi|^{1+i \rho} \int_{U} e^{-i[\xi, x]}|x|^{-1-i \rho} f(x) d x
$$

where $d x=d r d s$ and $d s$ is an invariant measure on hyperboloid $S_{H}(r)$.

Note that hyperbolic Fourier transform $\mathscr{F}_{h}^{\rho}$ is dependent on $\rho$; the reason is that this transform acts on its "own" component $\mathfrak{G S}^{n \sigma}$ (i.e., for $\sigma=-(n-2) / 2+i \rho$ ) simply as a scalar operator (see Corollary 11 from Proposition 10). Perhaps, uniform integral operator, acting on all subspaces $\mathfrak{G}^{n \sigma}$ as a scalar and invariant under $R(g)$, does not exist.

Proposition 8. The hyperbolic Fourier transform $\mathscr{F}_{h}^{\rho}$ is an intertwining operator of quasiregular representation $R$ in $L_{l o c}^{1}(U)$.

Proof. By definition of quasiregular representation and hyperbolic Fourier transform, we have

$$
\begin{aligned}
& {\left[\mathscr{F}_{h}^{\rho} \circ R(g) f\right](\xi)} \\
& \quad=\mathscr{F}_{h}^{\rho}(R(g) f)(\xi)
\end{aligned}
$$

$$
\begin{aligned}
& =|\xi|^{1+i \rho} \int_{U} e^{-i[x, \xi]}|x|^{-1-i \rho}(R(g) f)(x) d x \\
& =|\xi|^{1+i \rho} \int_{U} e^{-i[x, \xi]}|x|^{-1-i \rho} f\left(g^{-1} x\right) d x .
\end{aligned}
$$

On the other hand,

$$
\begin{aligned}
R & {\left[(g) \circ \mathscr{F}_{h}^{\rho} f\right](\xi) } \\
& =R(g)\left(\mathscr{F}_{h}^{\rho} f\right)(\xi) \\
& =R(g)\left(|\xi|^{1+i \rho} \int_{U} e^{-i[x, \xi]}|x|^{-1-i \rho} f(x) d x\right) \\
& =|\xi|^{1+i \rho} \int_{U} e^{-i\left[x, g^{-1} \xi\right]}|x|^{-1-i \rho} f(x) d x \\
& =|\xi|^{1+i \rho} \int_{U} e^{-i[g x, \xi]}|x|^{-1-i \rho} f(x) d x .
\end{aligned}
$$

Change variable $g x=t$. Then, $x=g^{-1} t$ and $d x=d t$ since the measure on $U$ is invariant under action of Lie group $\mathrm{SO}_{0}(n-$ $1,1)$. We have

$$
\begin{aligned}
& {\left[R(g) \circ \mathscr{F}_{h}^{\rho} f\right](\xi)} \\
& \quad=|\xi|^{1+i \rho} \int_{U} e^{-i[t, \xi]}\left|g^{-1} t\right|^{-1-i \rho} f\left(g^{-1} t\right) d t \\
& \quad=|\xi|^{1+i \rho} \int_{U} e^{-i[t, \xi]}|t|^{-1-i \rho} f\left(g^{-1} t\right) d t .
\end{aligned}
$$

So, $\mathscr{F}_{h}^{\rho} \circ R(g)=R(g) \circ \mathscr{F}_{h}^{\rho}$; that is, $\mathscr{F}_{h}^{\rho}$ is an intertwining operator.

Proposition 9. Let $f(x)$ be a pseudoradial function belonging to the space $L_{l o c}^{1}(U)$; that is, $f(x)=f_{0}\left([x, x]^{1 / 2}\right)$ for almost all $x \in U$. Then its integral transform $\mathscr{F}_{h}^{\rho}$ is pseudoradial for all $\xi \in U$ :

$$
\left[\mathscr{F}_{h}^{\rho} f\right](\xi)=F_{0}\left([\xi, \xi]^{1 / 2}\right)
$$

where

$$
\begin{aligned}
F_{0}(s)= & (-\pi i)^{(n-2) / 2} \cdot 2^{n / 2} \cdot s^{-(n-4) / 2+i \rho} \\
& \times \int_{0}^{+\infty} f_{0}(r) r^{(n-2) / 2-i \rho} K_{(n-2) / 2}(i r s) d r .
\end{aligned}
$$

Proof. Let $\widehat{f}(\xi)=\left(\mathscr{F}_{h}^{\rho} f\right)(\xi)$. Then, taking use of Proposition 8,

$$
\begin{aligned}
{[R(g) \widehat{f}](\xi) } & =\left(R(g) \circ \mathscr{F}_{h}^{\rho} f\right)(\xi) \\
& =\left(\mathscr{F}_{h}^{\rho} \circ R(g) f\right)(\xi) \\
& =\left(\mathscr{F}_{h}^{\rho} f\right)(\xi)=\widehat{f}(\xi) .
\end{aligned}
$$

We take into account that $R(g) f=f$ because $f$ is pseudoradial.

This proves the first part of the proposition. 
We fix now $\sqrt{[x, x]}=r$ and $\sqrt{[\xi, \xi]}=s$. Introduce the Euler coordinates on hyperboloids $S_{H}(r)$ and $S_{H}(s)$ :

$$
\begin{aligned}
x_{1} & =r \sinh \theta_{n-1} \sin \theta_{n-2} \cdot \ldots \cdot \cos \theta_{1}, \\
x_{2} & =r \sinh \theta_{n-1} \sin \theta_{n-2} \cdot \ldots \cdot \sin \theta_{1}, \\
& \vdots \\
x_{n-1} & =r \sinh \theta_{n-1} \cos \theta_{n-2}, \\
x_{n} & =r \cosh \theta_{n-1}, \\
\xi_{1} & =s \sinh \varphi_{n-1} \sin \varphi_{n-2} \cdot \ldots \cdot \cos \varphi_{1}, \\
\xi_{2} & =s \sinh \varphi_{n-1} \sin \varphi_{n-2} \cdot \ldots \cdot \sin \varphi_{1}, \\
& \vdots \\
\xi_{n-1} & =s \sinh \varphi_{n-1} \cos \varphi_{n-2}, \\
\xi_{n} & =s \cosh \varphi_{n-1} \cdot
\end{aligned}
$$

By definition of hyperbolic Fourier transform,

$$
\begin{aligned}
& {\left[\mathscr{F}_{h}^{\rho} f\right](\xi)} \\
& =|\xi|^{1+i \rho} \int_{0}^{+\infty} \int_{S_{H}(r)} e^{-i[x, \xi]}|x|^{-1-i \rho} f_{0}(\sqrt{[x, x]}) d r d s \\
& =|\xi|^{1+i \rho} \int_{0}^{+\infty} f_{0}(r) r^{n-1} r^{-1-i \rho} d r \\
& \quad \cdot \int_{S_{H}(r)} e^{-i\left[r x^{\prime}, s \xi^{\prime}\right]} \sinh ^{n-2} \theta_{n-1} \\
& \quad \cdot \sin ^{n-3} \theta_{n-2} \cdot \ldots \cdot \sin \theta_{2} d \theta_{n-1} d \theta_{n-2} \cdots d \theta_{1},
\end{aligned}
$$

where $x^{\prime}, \xi^{\prime} \in S_{H}$. Consider the inner integral $I$ on hyperboloid in more detail:

$$
\begin{aligned}
I=\int_{0}^{\infty} \int_{S^{n-2}} e^{-i r s \cosh \theta_{n-1} \cosh \varphi_{n-1}} & \\
& \times e^{i r s \sinh \theta_{n-1} \sinh \varphi_{n-1}\left\langle x^{\prime \prime}, \xi^{\prime \prime}\right\rangle} \\
& \cdot \sinh ^{n-2} \theta_{n-1} \sin ^{n-3} \theta_{n-2} \\
& \cdot \ldots \cdot \sin \theta_{2} d \theta_{n-1} \cdots d \theta_{1}
\end{aligned}
$$

where $x^{\prime \prime}$ and $\xi^{\prime \prime}$ belong to spheres, which are intersections of hyperboloids $[x, x]=r^{2}$ and $[\xi, \xi]=s^{2}$ by hyperplanes $x_{n}=r \cosh \theta_{n-1}$ and $\xi_{n}=s \cosh \varphi_{n-1}$ correspondingly:

$$
\begin{gathered}
I=\int_{0}^{\infty} e^{-i r s \cosh \theta_{n-1} \cosh \varphi_{n-1} \sinh ^{n-2} \theta_{n-1} d \theta_{n-1}} \\
\cdot \int_{S^{n-2}} e^{i r s \sinh \theta_{n-1} \sinh \varphi_{n-1}\left\langle x^{\prime \prime}, \xi^{\prime \prime}\right\rangle} \sin ^{n-3} \theta_{n-2} \\
\cdot \ldots \cdot \sin \theta_{2} d \theta_{n-2} \cdots d \theta_{1} .
\end{gathered}
$$

We calculate the inner integral on sphere in a standard way: first we integrate on a parallel $\left\langle x^{\prime \prime}, \xi^{\prime \prime}\right\rangle=\cos a$, orthogonal to vector $\xi^{\prime \prime}$; then we integrate by $a$ the obtained function in variable $a, 0 \leqslant a \leqslant \pi$ :

$$
\begin{aligned}
& \int_{S^{n-2}} e^{i r s \sinh \theta_{n-1} \sinh \varphi_{n-1} \cos a} d S \\
& =\int_{0}^{\pi} e^{i r s \sinh \theta_{n-1} \sinh \varphi_{n-1} \cos a}\left(\frac{2 \pi^{(n-2) / 2}}{\Gamma((n-2) / 2)}\right)(\sin a)^{n-3} d a,
\end{aligned}
$$

where $\left(2 \pi^{(n-2) / 2} / \Gamma((n-2) / 2)\right)(\sin a)^{n-3}$ is area of surface of $(n-3)$-dimensional sphere with radius $\sin a$.

After changing variables $\cos a=t, \sin a=\sqrt{1-t^{2}}$, we get

$$
\begin{aligned}
& \int_{S^{n-2}} e^{i r s \sinh \theta_{n-1} \sinh \varphi_{n-1} \cos a} d S \\
&=\frac{2 \pi^{(n-2) / 2}}{\Gamma((n-2) / 2)} \int_{-1}^{1} e^{i r s \sinh \theta_{n-1} \sinh \varphi_{n-1} \cdot t} \\
&=\frac{2 \pi^{(n-2) / 2}}{\Gamma((n-2) / 2)} \cdot \frac{\Gamma\left(1-t^{2}\right)^{(n-4) / 2} d t}{\left(-r s \sinh \theta_{n-1} \sinh \varphi_{n-1} / 2\right)^{(n-3) / 2}} \\
& \\
& \cdot \frac{\left(-r s \sinh \theta_{n-1} \sinh \varphi_{n-1} / 2\right)^{(n-3) / 2}}{\Gamma((n-2) / 2) \Gamma(1 / 2)} \\
&=\frac{(2 \pi)^{(n-2) / 2}}{\Gamma((n-2) / 2)} \cdot \frac{\Gamma((n-2) / 2) \Gamma(1 / 2)}{\left(-r s \sinh \theta_{n-1} \sinh \varphi_{n-1}\right)^{(n-3) / 2}} \\
& \quad \cdot J_{(n-3) / 2}\left(-r s \sinh \theta_{n-1} \sinh \varphi_{n-1}\right) t\left(1-t^{2}\right)^{(2((n-3) / 2)-1) / 2} d t \\
&=\left.\frac{(2 \pi)^{(n-1) / 2}}{\left(-r s \sinh \varphi_{n-1}\right)} \sinh \varphi_{n-1}\right)^{(n-3) / 2} \\
& \times J_{(n-3) / 2}\left(-r s \sinh \theta_{n-1} \sinh \varphi_{n-1}\right) \cdot
\end{aligned}
$$

Putting the found value of integral on sphere into the expression of hyperbolic Fourier transform, we get:

$$
\begin{aligned}
{\left[\mathscr{F}_{h}^{\rho} f\right](\xi)=} & \frac{(2 \pi)^{(n-1) / 2} s^{1+i \rho}}{\left(-s \sinh \varphi_{n-1}\right)^{(n-3) / 2}} \int_{0}^{+\infty} \frac{f_{0}(r) r^{n-1}}{r^{(n-3) / 2}} r^{-1-i \rho} \\
& \cdot \int_{0}^{+\infty} e^{-i r s \cosh \theta_{n-1} \cosh \varphi_{n-1}} \\
& \quad \times \sinh ^{(n-1) / 2} \theta_{n-1} \\
& \quad \times J_{(n-3) / 2}\left(-r s \sinh \theta_{n-1} \sinh \varphi_{n-1}\right) d \theta_{n-1} d r .
\end{aligned}
$$


Change variables $z=\sinh \theta_{n-1}, \cosh \theta_{n-1}=\sqrt{1+z^{2}}$, and $d z=\sqrt{1+z^{2}} d \theta_{n-1}$, so we have

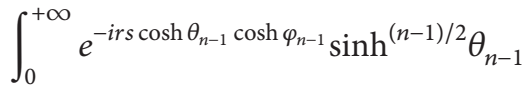

$$
\begin{aligned}
& \times J_{(n-3) / 2}\left(-r s \sinh \theta_{n-1} \sinh \varphi_{n-1}\right) d \theta_{n-1} \\
& =\int_{0}^{+\infty} \frac{e^{-i r s \cosh \varphi_{n-1} \sqrt{1+z^{2}}} z^{(n-1) / 2}}{\sqrt{1+z^{2}}} \\
& \cdot J_{(n-3) / 2}\left(-r s \sinh \varphi_{n-1} z\right) d z \text {. }
\end{aligned}
$$

Now we apply integral from [9], Section 2.12.10, formula (10), and set $\rho=\operatorname{irs} \cosh \varphi_{n-1}, z=1, x=z, c=-r s \sinh \varphi_{n-1}$, and $v=(n-3) / 2$. Finally we have

$$
\begin{aligned}
\int_{0}^{+\infty} & \frac{e^{-i r s \cosh \varphi_{n-1} \sqrt{1+z^{2}}} z^{(n-1) / 2}}{\sqrt{1+z^{2}}} \\
& \cdot J_{(n-3) / 2}\left(-r s \sinh \varphi_{n-1} z\right) d z \\
= & \sqrt{\frac{2}{\pi}}\left(-r s \sinh \varphi_{n-1}\right)^{(n-3) / 2}(\text { irs })^{-(n-2) / 2} K_{(n-2) / 2}(\text { irs }) .
\end{aligned}
$$

Hence,

$$
\begin{aligned}
{\left[\mathscr{F}_{h}^{\rho} f\right](\xi)=} & 2^{n / 2} \pi^{(n-2) / 2} \\
& \times s^{1+i \rho} \int_{0}^{+\infty} f_{0}(r) r^{n-1} r^{-1-i \rho} \\
& \cdot(i r s)^{-(n-2) / 2} K_{(n-2) / 2}(i r s) d r \\
= & (-\pi i)^{(n-2) / 2} 2^{n / 2} s^{-(n-4) / 2+i \rho} \\
& \times \int_{0}^{\infty} f_{0}(r) r^{(n-2) / 2-i \rho} K_{(n-2) / 2}(i r s) d r .
\end{aligned}
$$

Proposition 9 is proved.

Proposition 10. Suppose function $f(x) \in L_{\text {loc }}^{1}(U)$ is a product of pseudoradial function and space hyperbolic harmonic of homogeneity degree $\sigma$ :

$$
f(x)=f_{0}\left([x, x]^{1 / 2}\right) \widetilde{H}_{L}^{n, \sigma}(x),
$$

and then its $\mathscr{F}_{h}^{\rho}$-transform is

$$
\left[\mathscr{F}_{h}^{\rho} f\right](\xi)=F^{n, \sigma}\left([\xi, \xi]^{1 / 2}\right) \widetilde{H}_{L}^{n, \sigma}(\xi),
$$

where

$$
\begin{aligned}
F^{n, \sigma}(s)= & 2^{n / 2}(-\pi i)^{(n-2) / 2} \\
& \cdot s \int_{0}^{+\infty} f_{0}(r) K_{\sigma+(n-2) / 2}(i r s) d r .
\end{aligned}
$$

Proof. We have, by definition of hyperbolic Fourier transform,

$$
\begin{aligned}
{\left[\mathscr{F}_{h}^{\rho} f\right](\xi)=|\xi|^{1+i \rho} \int_{U} e^{-i[x, \xi]}|x|^{-1-i \rho} f_{0}(\sqrt{[x, x]}) \widetilde{H}_{L}^{n \sigma}(x) d x } \\
=|\xi|^{1+i \rho} \int_{0}^{+\infty} f_{0}(r) r^{-1-i \rho} \\
\quad \times\left(\int_{S_{H}(r)} e^{-i[x, \xi]} \widetilde{H}_{L}^{n \sigma}(x) d x\right) d r .
\end{aligned}
$$

Let $x=r x^{\prime}, \xi=s \xi^{\prime}$, where $x^{\prime}, \xi^{\prime} \in S_{H}$. Because $\widetilde{H}_{L}^{n \sigma}$ is a homogeneous function of homogeneity degree $\sigma$,

$$
\widetilde{H}_{L}^{n \sigma}(x)=\widetilde{H}_{L}^{n \sigma}\left(r x^{\prime}\right)=r^{\sigma} H_{L}^{n \sigma}\left(x^{\prime}\right) .
$$

Hence,

$$
\begin{aligned}
& {\left[\mathscr{F}_{h}^{\rho} f\right](\xi)} \\
& =|\xi|^{1+i \rho} \int_{U} e^{-i[x, \xi]}|x|^{-1-i \rho} f_{0}(\sqrt{[x, x]}) \widetilde{H}_{L}^{n \sigma}(x) d x \\
& \text { (we change variable } x=r x^{\prime}, d x=r^{n-1} d x^{\prime} \text { ) } \\
& =|\xi|^{1+i \rho} \int_{0}^{+\infty} f_{0}(r) r^{-1-i \rho} \\
& \times\left(\int_{S_{H}} e^{-i r s\left[x^{\prime}, \xi^{\prime}\right]} r^{\sigma} H_{L}^{n \sigma}\left(x^{\prime}\right) r^{n-1} d x^{\prime}\right) d r \\
& =|\xi|^{1+i \rho} \int_{0}^{+\infty} f_{0}(r) r^{\sigma+n-1} r^{-1-i \rho} \\
& \times\left(\int_{S_{H}} e^{-i r s\left[x^{\prime}, \xi^{\prime}\right]} H_{L}^{n \sigma}\left(x^{\prime}\right) d x^{\prime}\right) d r .
\end{aligned}
$$

We make use of formula (32) from [6] to calculate the integral on $S_{H}$. Namely, for each $\xi^{\prime} \in S_{H}$, the equality takes place:

$$
\begin{aligned}
\int_{S_{H}} e^{-i r s\left[x^{\prime}, \xi^{\prime}\right]} H_{L}^{n \sigma}\left(x^{\prime}\right) d x^{\prime} \\
\quad=2^{n / 2}\left(-\frac{\pi i}{r s}\right)^{(n-2) / 2} K_{\sigma+(n-2) / 2}(\text { irs }) H_{L}^{n \sigma}\left(\xi^{\prime}\right) .
\end{aligned}
$$

Now we have

$$
\begin{aligned}
{\left[\mathscr{F}_{h}^{\rho} f\right](\xi)=} & 2^{n / 2} s^{1+i \rho} H_{L}^{n \sigma}\left(\xi^{\prime}\right) \\
& \cdot \int_{0}^{+\infty} f_{0}(r) r^{\sigma+n-1} r^{-1-i \rho} \\
& \times\left(-\frac{\pi i}{r s}\right)^{(n-2) / 2} K_{\sigma+(n-2) / 2}(i r s) d r \\
= & 2^{n / 2} \widetilde{H}_{L}^{n \sigma}(\xi) s \cdot(-\pi i)^{(n-2) / 2} \\
& \times \int_{0}^{+\infty} f_{0}(r) K_{\sigma+(n-2) / 2}(i r s) d r \\
= & F^{n \sigma} \widetilde{H}_{L}^{n \sigma}(\xi),
\end{aligned}
$$


where

$$
\begin{aligned}
F^{n \sigma}(s)= & 2^{n / 2} \widetilde{H}_{L}^{n \sigma}(\xi) s \cdot(-\pi i)^{(n-2) / 2} \\
& \times \int_{0}^{+\infty} f_{0}(r) K_{\sigma+(n-2) / 2}(i r s) d r
\end{aligned}
$$

Proposition 10 is proved.

Corollary 11. If $\sigma=-(n-2) / 2+i \rho$, then hyperbolic Fourier transform $\mathscr{F}_{h}^{\rho}$ acts on the space $\mathfrak{H}^{n \sigma}$ of space hyperbolic harmonics as a scalar operator:

$$
\left[\mathscr{F}_{h}^{\rho} \widetilde{H}_{L}^{n \sigma}\right](\xi)=\lambda_{n \sigma} \widetilde{H}_{L}^{n \sigma}(\xi),
$$

where

$$
\lambda_{n \sigma}=\frac{(-2 \pi i)^{n / 2}}{2 \cosh (\pi \rho / 2)} .
$$

Proof. Consider $\left[\mathscr{F}_{h}^{\rho} \widetilde{H}^{n \sigma}\right](\xi)$. Let us use Proposition 10. Because $f_{0}\left([\xi, \xi]^{1 / 2}\right) \equiv 1$, we have

$$
\begin{aligned}
{\left[\mathscr{F}_{h}^{\rho} \widetilde{H}^{n \sigma}\right](\xi)=} & 2^{n / 2}(-\pi i)^{(n-2) / 2} s \widetilde{H}^{n \sigma}(\xi) \\
& \times \int_{0}^{+\infty} K_{\sigma+(n-2) / 2}(i r s) d r .
\end{aligned}
$$

We apply the integral from [9], Section 2.16.2, formula (1), and set $v=i \rho$ and $c=i$ s. Finally we have (this integral should be understood in a regularized value sense too) the following:

$$
\begin{aligned}
& {\left[\mathscr{F}_{h}^{\rho} \widetilde{H}^{n \sigma}\right](\xi)} \\
& \quad=2^{n / 2}(-\pi i)^{(n-2) / 2} \cdot s \cdot \frac{\pi}{2 i s} \cdot \frac{1}{\cos (i \pi \rho / 2)} \widetilde{H}^{n \sigma}(\xi) \\
& \quad=\frac{(-2 \pi i)^{n / 2}}{2 \cosh (\pi \rho / 2)} \widetilde{H}^{n \sigma}(\xi) .
\end{aligned}
$$

Corollary 11 is proved.

Corollary 12. The inverse hyperbolic Fourier transform $\left(\mathscr{F}_{h}^{\rho}\right)^{-1}$ on each of the spaces $\mathfrak{G S}^{n \sigma}$ has the next form:

$$
\begin{aligned}
& {\left[\left(\mathscr{F}_{h}^{\rho}\right)^{-1} f\right](\xi)} \\
& \quad=\frac{2 \cosh (\pi \rho / 2)}{(-2 \pi i)^{n / 2}}|\xi|^{1+i \rho} \int_{U} e^{i[\xi, x]}|x|^{-1-i \rho} f(x) d x .
\end{aligned}
$$

The proof evidently follows from Corollary 11.

Remark 13. A well-known theorem asserts that any intertwining operator of the quasiregular representation of a compact group is a convolution [5, chapter V, Section 2, Theorem 2.3]. However, the question whether this theorem is true for representation $R$ of Lie group $S_{0}(n-1,1)$ in $L_{\text {loc }}^{1}(U)$ is still open. Due to the exact sequence

$$
1 \longrightarrow S O(n-1) \longrightarrow S_{0}(n-1,1) \longrightarrow S_{H} \longrightarrow 1,
$$

any function $\varphi(x)$ defined on a Lobachevsky space $S_{H}$ could be raised to function $\widetilde{\varphi}$ on $G=S O_{0}(n-1,1)$ that is constant on the left cosets under subgroup $S O(n-1)$. An analog of this theorem in $L^{1} \cap L^{2}\left(S_{H}\right)$ is valid as it was shown in the author's paper [10]. Our proof method uses Fourier transform and an ordinary convolution of functions on $G$ :

$$
[\tilde{\varphi} * \tilde{\psi}](x)=\int_{G} \tilde{\varphi}(g) \widetilde{\psi}\left(g^{-1} x\right) d g .
$$

We hope that the technique developed in this work (including the hyperbolic Fourier transform) will be able to prove that intertwining operators of quasiregular representation of Lorentz group are also involutions in interior of the light cone.

\section{Conflict of Interests}

The author declares that there is no conflict of interests regarding the publication of this paper.

\section{References}

[1] E. M. Stein and G. Weiss, Introduction to Fourier analysis on Euclidean Spaces, Princeton University Press, 1971.

[2] N. Y. Vilenkin, Special Functions and Representations of Groups, Nauka, Moscow, Russia, 2nd edition, 1991.

[3] I. M. Gel'fand, M. I. Graev, and N. Y. Vilenkin, Integral Geometry and Representation Theory, Generalized Functions, vol. 5, Academic Press, New York, NY, USA, 1966.

[4] V. P. Burskii and T. V. Shtepina, "On the spectrum of an equivariant extension of the Laplace operator in a ball," Ukrainian Mathematical Journal, vol. 52, no. 11, pp. 1679-1690, 2000.

[5] S. Helgason, Groups and Geometric Aanalysis, vol. 113, Academic Press, 1984.

[6] V. V. Shtepin and T. V. Shtepina, "An application of intertwining operators in functional analysis," Izvestiya Mathematics, vol. 73, no. 6, pp. 1265-1288, 2009.

[7] A. Erdélyi, "Die Funksche Integralgleichung der Kugelflächenfunktionen und ihre Übertragung auf die Überkugel," Mathematische Annalen, vol. 115, no. 1, pp. 456-465, 1938.

[8] T. V. Shtepina, "A generalization of the Funk-Hecke theorem to the case of hyperbolic space," Izvestiya: Mathematics, vol. 68, no. 5, pp. 1051-1061, 2004.

[9] A. P. Prudnikov, Y. A. Brychkov, and O. I. Marichev, Integrals and Series: Special Functions, vol. 2, Gordon \& Breach, New York, NY, USA, 1990.

[10] T. V. Shtepina, "About representation as convolution of the operator, permutable with the operator quasiregular representations of group of Lorentz,' Trudy Instituta Prikladnoj Matematiki i Mekhaniki, vol. 7, pp. 225-228, 2002. 


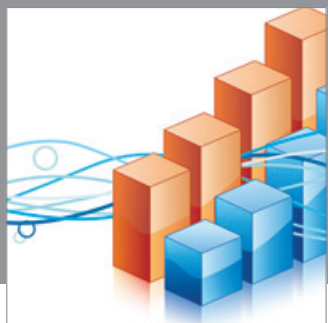

Advances in

Operations Research

mansans

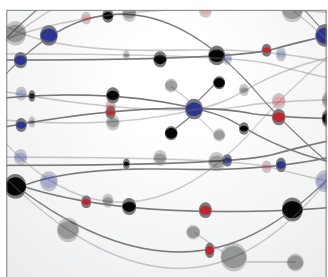

The Scientific World Journal
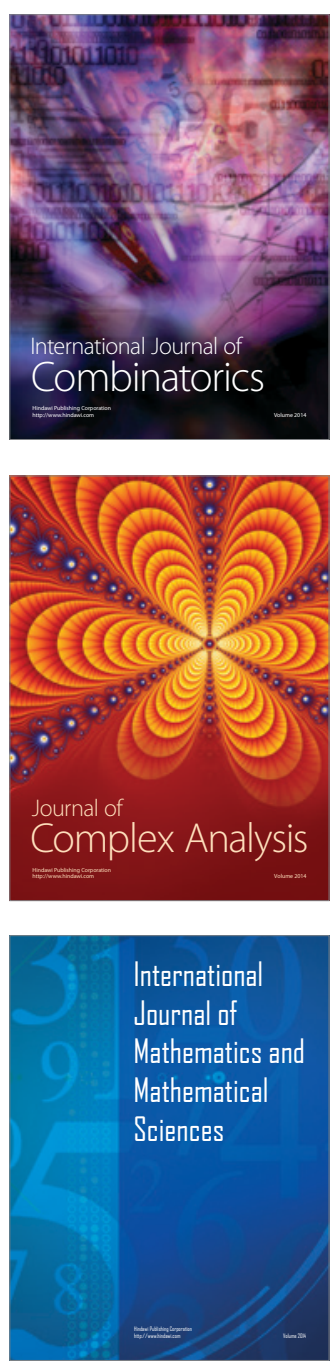
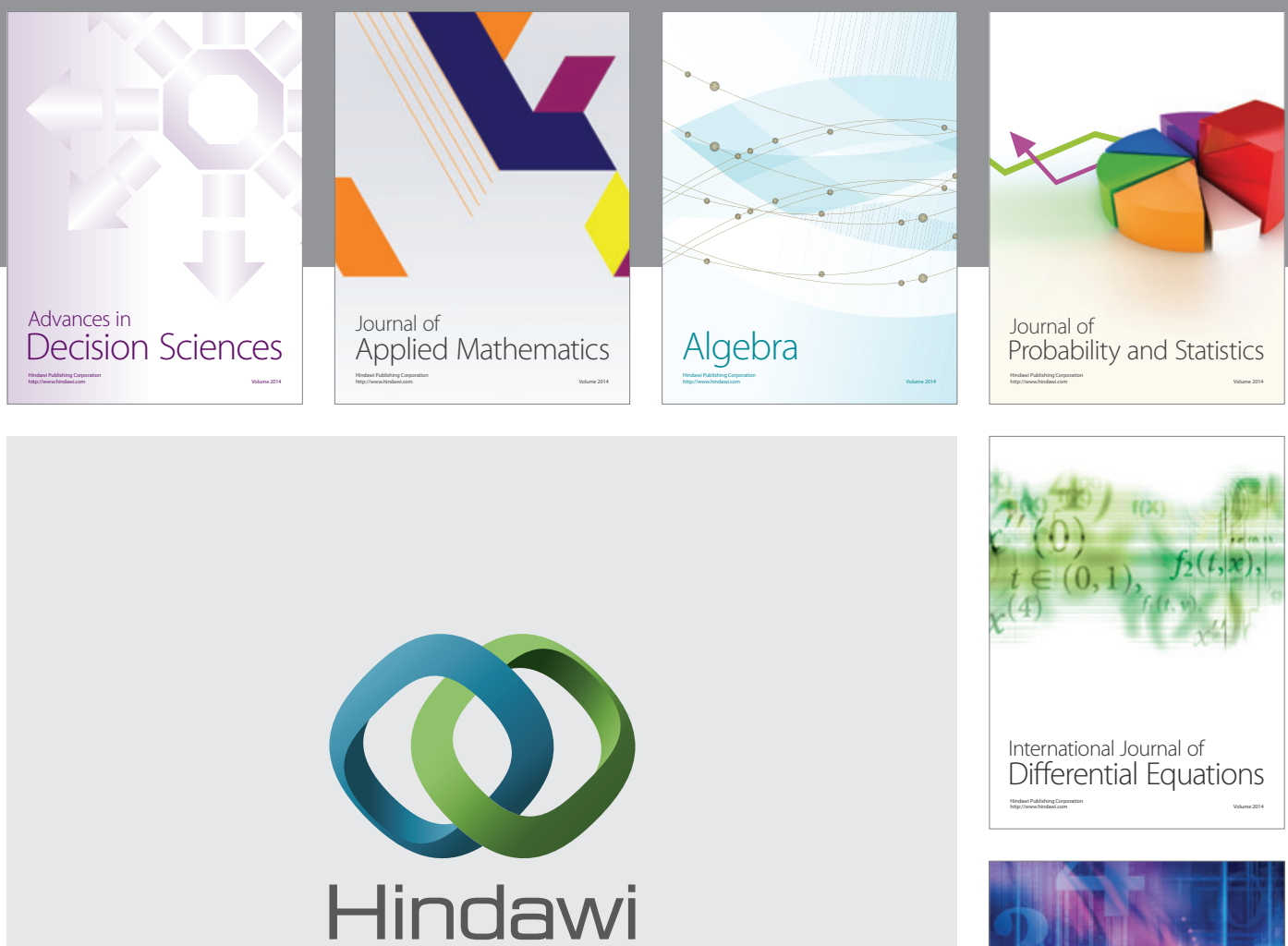

Submit your manuscripts at http://www.hindawi.com
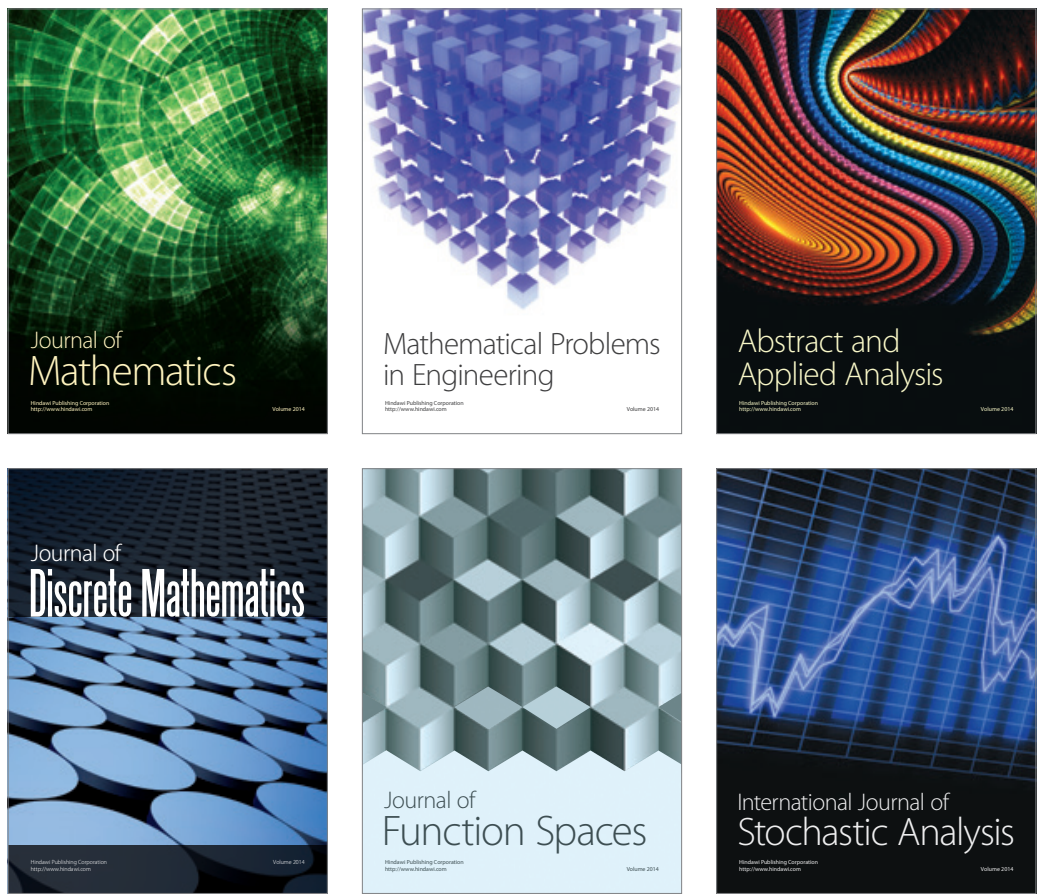

Journal of

Function Spaces

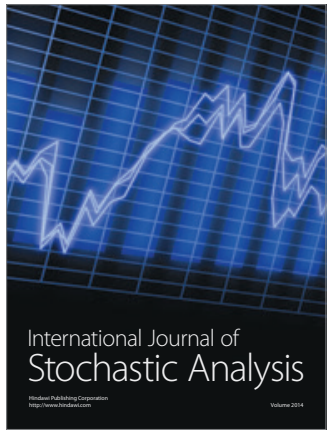

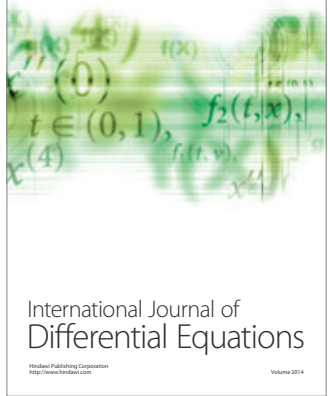
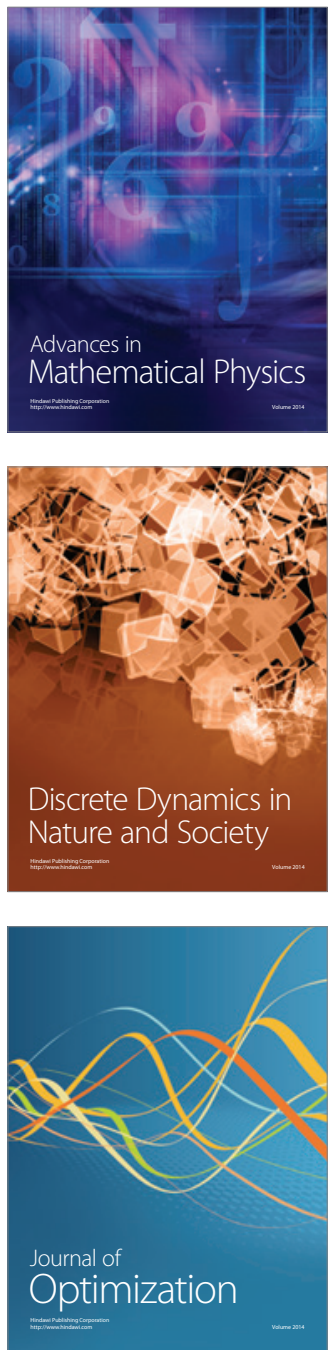